\title{
Entwicklung eines bullösen Pemphigoids unter Efalizumab-Therapie bei Psoriasis vulgaris
}

\section{Occurence of a Bullous Pemphigoid with Efalizumab Therapy in Psoriasis Vulgaris}

Autoren

Institut
F. Oberthür, S. Baur-Beger, A. Wagner, C. Metzen, C. Bayerl

Klinik für Dermatologie und Allergologie, Städtisches Klinikum Wiesbaden, HSK, Wilhelm-Fresenius-Klinik GmbH, Lehrkrankenhaus der Universität Mainz

\section{Bibliografie}

DOI $10.1055 / \mathrm{s}-2007-995544$

Akt Dermatol 2008; 34:

19-21 ๑ Georg Thieme

Verlag KG Stuttgart · New York ISSN 0340-2541

Korrespondenzadresse

\section{Frank Oberthür}

Klinik für Dermatologie und Allergologie, Städtisches Klinikum Wiesbaden HSK, Wilhelm-Fresenius-Klinik GmbH, Lehrkrankenhaus der Universität Mainz, Aukammallee 39 65191 Wiesbaden Frank-Oberthuer@gmx.net

\section{Zusammenfassung \\ $\nabla$}

Bei einer 85-jährigen Patientin mit einer seit dem 20. Lebensjahr bekannten Psoriasis vulgaris kam es vier Wochen nach Einleitung einer Therapie mit Efalizumab zur Entwicklung eines bullösen Pemphigoids. Nach dem Absetzen von Efalizumab und der Einleitung einer systemischen Ste-

\section{Anamnese \\ $\nabla$}

Unsere 85-jährige Patientin litt seit dem 20. Lebensjahr an einer chronisch-rezidivierenden Psoriasis vulgaris, welche in den letzten Wochen zur Generalisation neigte. Davor bestand über mehrere Jahre Erscheinungsfreiheit. Dennoch hatte sie systemische und PUVA-Therapien in den Jahren ihrer Erkrankung bereits erhalten. Da der jetzige Schub in der ambulanten Behandlung mit oralen und topischen Steroiden nicht zu bessern war, wurde 7 Wochen vor Erstvorstellung in unserer Klinik eine Therapie mit Efalizumab einmal wöchentlich s.c. eingeleitet. Nach deutlichem Ansprechen der Psoriasis auf diese Therapie entwickelte sich nach insgesamt 4 Wochen ein juckendes Exanthem mit Bullae, zunächst nur an Handrücken, Hüften und am Rücken. Im weiteren Verlauf kam es zur Ausbreitung auf das gesamte Integument. Krankheitsgefühl, Fieber oder Nachtschweiß bestanden nicht. Ein vorausgegangener Infekt war nicht eruierbar.

\section{Lokalbefund}

\section{$\nabla$}

Am gesamten Integument inklusive Capillitium, Palmae und Plantae fanden sich multiple indurierte Erytheme, vereinzelt mit prallen bis zu mehreren Zentimetern großen Blasen. Am unteren Rücken und glutaeal bestanden blasige Abhebungen der Haut, z.T. auch Krusten ( $\bullet$ Abb. 1). roidtherapie in Kombination mit Azathioprin kam es zu einer raschen Abheilung der bullösen Dermatose. Auch nach Beendigung der Steroidund Azathioprin-Therapie blieb die Patienten seit nunmehr 8 Monaten beschwerdefrei. Efalizumab muss daher in dieser Kasuistik als Auslöser des bullösen Pemphigoids angesehen werden.

An den Plantae beidseits fanden sich zusätzlich multiple, stecknadelkopfgroße hämorrhagische Blasen. Die Psoriasis war zum Zeitpunkt der Aufnahme unter Efalizumab komplett abgeheilt.

\section{Laborbefunde}

$\nabla$

BSG 32/71 mm n.W.; Leukozyten 18,6/nl; Lymphozyten 15,7\%; Eosinophile 9,9\%;. Kreatinin $1,5 \mathrm{mg} / \mathrm{dl}$, Kreatinin-Clearance $44 \mathrm{ml} / \mathrm{min}$, Harnsäure $7,6 \mathrm{mg} / \mathrm{dl}$, CRP $1,6 \mathrm{mg} / \mathrm{dl}$. TSH basal $5,16 \mu \mathrm{IU} / \mathrm{ml}$, erhöht; FT3 $1,96 \mathrm{pg} / \mathrm{ml}$, erniedrigt; FT4 normwertig. Die übrigen Laborpararmeter der klinischen Chemie und des Differenzialblutbildes sowie die Serum-Eiweißelektrophorese waren unauffällig.

Auto-Antikörper: ANA negativ. Pemphigus-Antikörper: Negativ. Pemphigoid-Antikörper: 1:1280 positiv.

Urinstatus: Spezifisches Gewicht 1,015, Protein im Urin schwach positiv, Blut im Urin schwach positiv, ansonsten unauffällig.

\section{Weitere Untersuchungsbefunde} $\nabla$

Sonografie des Abdomens, Röntgen Thorax und gynäkologische Untersuchung unauffällig.

Dermato-Histologie: In der Biopsie zeigt sich eine subepidermale Blasenbildung. Am Blasenboden finden sich Epithelreste sowie Entzün- 


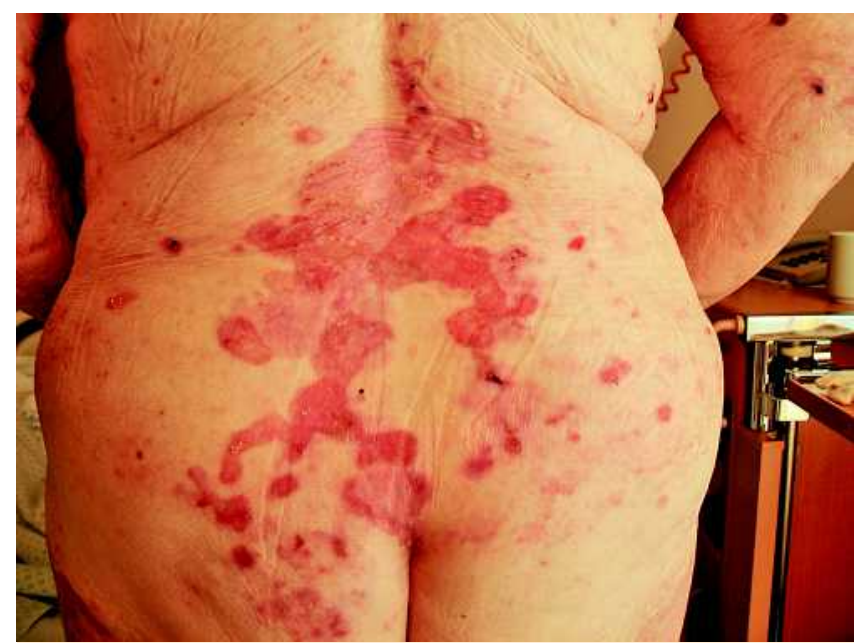

Abb. 1 Dermatohistopathologie: Subepidermale Blasenbildung mit Abhebung der unveränderten Epidermis.

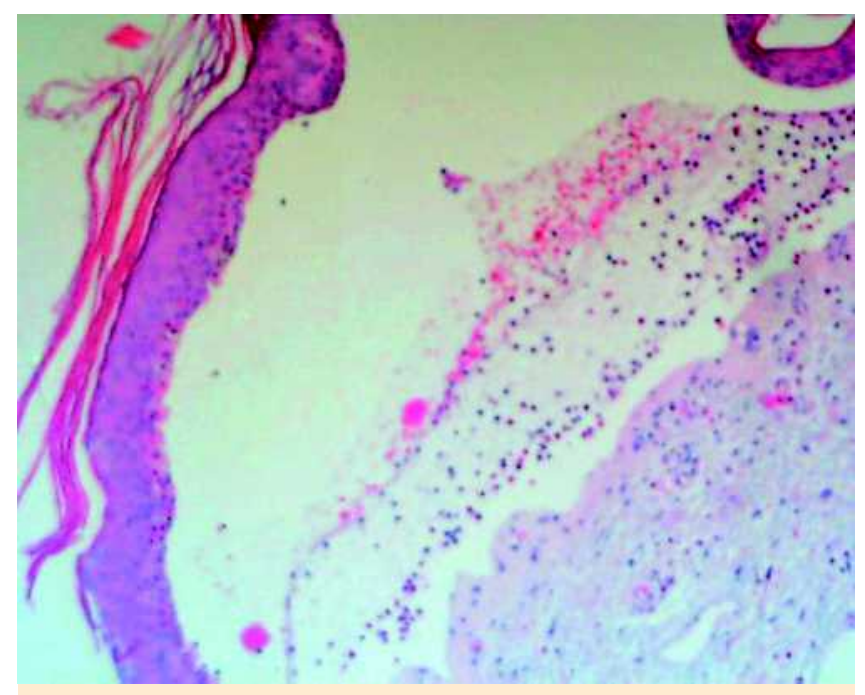

Abb. 2 Multiple indurierte Erytheme mit flachen Blasen und Erosionen.

dungszellen (eosinophile und neutrophile Granulozyten, Lymphozyten). Die Epidermis weist eine Spongiose auf. Im Randbereich besteht eine ödematöse Schwellung der Papillarkörper. Im oberen Korium findet sich ein entzündliches Infiltrat aus neutrophilen und eosinophilen Granulozyten. Ein mit einem bullösen Pemphigoid vereinbarer Befund ( $\bullet$ Abb. 2).

In der Immunfluoreszenz zeigte sich eine deutliche bandförmige Fluoreszenz entlang der Basalmembranzone für Antihuman-C3 und -IgG. Für Antihuman-IgA und -IgM war keine spezifische Fluoreszenz nachweisbar.

\section{Therapie und Verlauf}

Die Therapie mit Efalizumab wurde unverzüglich beendet. Systemisch verordneten wir Prednisolon initial $60 \mathrm{mg}$ in ausschleichender Dosierung in Kombination mit Azathioprin 100 mg/die. Die topische Therapie erfolgte im Bereich des Capillitiums mit Betamethason $2 \times /$ die, die der Körperherde mit 0,1\% Betamethason $-2 \%$ Triclosan in Vaseline. Nach Ansprechen der Behandlung wurde auf $1 \%$ Hydrocortison - $2 \%$ Triclosan in Unguentum
Cordes $2 \times /$ die umgestellt. Die Lokaltherapie bei Entlassung erfolgte mit $2 \%$ Triclosan in Unguentum Cordes.

Unter dieser Therapie kam es zu einer raschen Besserung und Abheilung des Lokalbefundes. Auch nach Beendigung der Steroid- und Azathioprintherapie ist die Patientin nun schon seit insgesamt acht Monaten erscheinungsfrei, auch bezüglich der Psoriasis.

\section{Diskussion \\ $\nabla$}

Biologicals greifen gezielt in die Pathogenese der Psoriasis ein. Efalizumab ist in Europa zur Behandlung der mittelschweren bis schweren chronischen Psoriasis vom Plaque-Typ zugelassen. Efalizumab ist ein rekombinierter, humaner, monoklonaler IgG1-Antikörper, der an die CD11A-Untereinheit des LFA-1-Liganden auf der T-Zelle bindet. Es ist ein selektives Immunsuppressivum, das die Aktion der T-Lymphozyten beeinflusst. Vorteile gegenüber chemisch hergestellten Arzneimitteln bestehen im Fehlen von Organtoxizitäten und Arzneimittelinterferenzen. Beachtet werden muss vor allem das Infektionsrisiko.

Zum bekannten Nebenwirkungsprofil gehört u. a. eine mögliche Reaktivierung von latenten chronischen Infektionen. Ebenso wurde über Thrombozytopenien sowie in Einzelfällen über schwere hämolytische Anämien während der Behandlung mit Efalizumab berichtet. Beobachtet wurden ferner Fälle entzündlicher Polyradikuloneuropathien, Fälle von Arthritis sowie Exazerbationen der Psoriasis. Die Entwicklung eines bullösen Pemphigoids unter Efalizumabtherapie wurde bislang nicht beschrieben.

In der Literatur finden sich über 40 Fälle von blasenbildenden Dermatosen bei Psoriasis Patienten, wobei es meistens zur Entwicklung eines bullösen Pemphigoids kam, insbesondere nach einer UVB $311 \mathrm{~nm}$ oder Psoralen-UVA Therapie [1]. Wiederholt wurde über eine Medikamenten-induzierte Blasenbildung z.B. durch Angiotensin-II-Antagonisten oder Etanercept berichtet $[3,6]$; aber auch über ein spontanes Auftreten von blasenbildenden Dermatosen bei Psoriasis vulgaris [2]. Ebenfalls 4 Wochen nach Efalizumab-Behandlung wurde in einer Kasuistik ein DRESS-Syndrom beschrieben mit der typischen suepidermalen Blasenbildung und eosinophilenreichem Zellinfiltrat [4]. Die genauen pathologischen Beziehungen zwischen Psoriasis vulgaris und bullösem Pemphigoid sind bisher unklar, meist wird als Auslöser jedoch eine Immunmodulation verantwortlich gemacht $[5,7]$.

Das Risiko der Entwicklung beziehungsweise der Verstärkung einer Autoimmunerkrankung unter einer Efalizumab-Therapie ist jedoch eher gering. Insbesondere im Vergleich mit den althergebrachten systemischen Antipsoriatika wie Methotrexat, Cyclosporin, Acitretin oder Fumarsäure scheinen die neuen Biologicals aufgrund der bereits erwähnten fehlenden Organtoxizität deutlich überlegen. Beachtet werden muss, dass es bisher keine Langzeitdaten gibt. 


\section{Abstract}

\section{Occurence of a Bullous Pemphigoid with Efalizumab Therapy in Psoriasis Vulgaris}

$\nabla$

We report the case of an 85-year-old female patient suffering from psoriasis since her twenties who developed a bullous pemphigoid 4 weeks after the start of a therapy with efalizumab. Withdrawal of efalizumab-therapy in combination with the use of systemic steroids and azathioprine led to a rapid healing of the bullous pemphigoid. Even after the ending of the steroid and azathioprine therapy, there was no reactiviation of bullous pemphigoid and the patient has remained free of symptoms for 8 months now. Efalizumab seems to have been the causative drug inducing bullous pemphoid in our patient.

\section{Literatur}

1 Arregui MA, Soloeta R et al. Bullous pemphigoid related to PUVA therapy: Two further cases. Actas Dermosifiliogr 2006; 97: 444-7

2 Lazarczyk M, Wozniak Ket al. Coexistence of psoriasis and pemphigoid - only a coincidence? Int J Mol Med 2006; 18: 619-623

3 Saraceno R, Citarella L, Spalione G, Cimenti S. A biological approach in a patient with psoriasis and bullous pemphigoid associated with losartan therapy. Clin Exp Dermatol 2008; 33: 154- 155

4 White JM, Smith Ch, Robson A, Ash G, Barker JN. DRESS syndrome caused by efalizumab. Clin Exp Dermatol 2007; 19: 50-52

5 Wilczek A, Sticherling M. Concomitant psoriasis and bullous pemphigoid: coincidence or pathogenic relationship? Int J Dermatol 2006; 45: $1353-1357$

6 Yamauchi PS, Lowe NJ et al. Treatment of coexisting bullous pemphigoid and psoriasis with the tumor necrosis factor antagonist etanercept. J Am Acad Dermatol 2006; 54: 121 - 122

7 Yasuda H, Tomita Y, Shibaki A, Hashimoto T. Two cases of subepidermal blistering disease with anti-p200 or 180-kD bullous pemphigoid antigen associated with psoriasis. Dermatology 2004; 209: 149-155

\section{Buchbesprechung}

\section{Kontaktekzem}

T. Fuchs, W. Aberer (Hrsg)

Deisenhofen: Dustri-Verlag Dr. Karl Feistle, 2. Aufl. 2007. 700 S., 220 Abb., 100 Tab. Ringbuch/Loseblatt, 137,- $€$

ISBN Altauflage 2002: 3-87185-292-9

ISBN Neuauflage 2007: 978-3-87185-377-7

Seit der Erstauflage 2002 sind 5 Jahre vergangen und nun liegt die Zweitauflage des „Kontaktekzem-Ringbuchs“ von Prof. Fuchs, Göttingen und Prof. Aberer, Graz vor.

Hierin findet sich das gesammelte Wissen zum Kontaktekzem, zusammengefasst von 45 deutschsprachigen, erfahrenen Allergologen. Hauptanliegen der Herausgeber ist es, die neuen Erkenntnisse zur Auslösung des Kontaktekzems zu vermitteln, um damit die Aufklärungsquote von Kontaktallergien zu erhöhen und besser präventiv beraten zu können. Die 51 Kapitel beginnen mit einer aktuellen Einführung zur Historie des Kontaktekzems, zu den immunologischen Grundlagen und der Histologie, zur Hornschichtbarriere, zu Kontaktallergiemodellen, zu prädiktiven In-vitro-Testungen für Kontaktallergene und zur Epidemiologie. Bei all diesen vorgestellten Neuerungen bleibt die Empfehlung weiterhin nach den Leitlinien zum Kontaktekzem den Epikutantest durchzuführen, da kein praktikables Ersatzverfahren zur Verfügung steht. Unter Klinik und Differenzialdiagnose sind Arbeiten gesammelt zur irritativen Dermatitis, Kontaktallergien an verschiedenen Körperregionen und der Mundschleimhaut, dem phototoxischen und photoallergischen Kontaktekzem, dem irritativ provozierten atopischen Ekzem, der Proteinkontaktdermatitis, zur Kontakturtikaria und zu Differenzialdiagnosen des Kontaktekzems. Unter diesen Differenzialdiagnosen findet sich eine eindrucksvolle Abbildung eines extramammären Morbus Paget, der fälschlich zwei Jahre lang als Kontaktekzem behandelt wurde. Im größten und wichtigsten Buchabschnitt über die Noxen werden die häufigsten Allergengruppen und Einzelallergene vorgestellt. Gut, dass sich auch immer wieder die Strukturformeln finden lassen. Das Werk endet mit Kapiteln zu verschiedenen Altersgruppen und geschlechtsspezifischen Unterschieden des Kontaktekzems, zur Therapie und zur Klassifikation von Handekzemen. Das Sachwortregister, wichtig für ein Nachschlagewerk, erfüllt seinen Zweck, da es ausreichend ausführlich ist. Die Literatur nach jedem Kapitel umfasst die letzten 30 Jahre. Abbildungen und Tabellen sind gelungen und unterstützen damit den Text. Der Band ist hervorragend zum Nachschlagen. Der allergologisch tätige Dermatologe wird es sicher häufig aus dem Regal nehmen.

C. Bayerl, Wiesbaden 